\title{
Joint Pilot Allocation for Fronthaul and Access Links in Cloud Radio Access Networks
}

\author{
Zhendong Mao, Yuexia He and Ying Li
}

\begin{abstract}
Acquisition of instantaneous channel state information (CSI) is essential in cloud radio access networks (C-RANs) to achieve advanced technologies. However, the overhead to obtain CSIs by traditional schemes is sufficiently large, which will degrade the spectrum efficiency. To handle this problem, a two-phase based pilot allocation scheme is proposed in this paper, where both the wireless fronthaul link and radio access link are considered. In particular, the pilot allocation for RRH is derived based on the angular resolution characteristics in phase I. In phase II, an inter-cluster and intra-cluster pilot allocation algorithm for users is proposed to mitigate the pilot contamination. Numerical results are shown to demonstrate the performance of our proposed pilot allocation scheme in C-RANs.
\end{abstract}

\section{INTRODUCTION}

The fifth generation of mobile technology $(5 \mathrm{G})$ is proposed to deal with the increasing demands and business contexts recently [1] [2] [3]. The cloud radio access network (CRAN) is a promising solution to provide high energy and spectrum efficiencies, in which distributed remote radio heads (RRHs) connect to the baseband unit (BBU) pool through the wired/wireless fronthaul link. Several techniques are proposed to realize the advantages of C-RANs, such as large-scale collaborative processing and the radio resource allocation and optimization [4] [5] [6]. However, these works are assumed that the BBU pool has the knowledge of CSIs, which has to be estimated in practice.

Therefore, obtaining accurate instantaneous CSIs is of great significance in C-RANs. Since the wireless fronthaul technology is adapted in the real world for the advantages of cheapness and flexibility, both the radio access link between the users and RRHs and the wireless fronthaul link between the RRHs and the BBU pool need to be estimated, respectively [7] [8] [9].

In [7], a segment training based individual channel estimation is proposed in C-RANs. The sequential minimum meansquare-error (MMSE) estimator is proposed to estimate the Permission to make digital or hard copies of all or part of this work for 1 personal or classroom use is granted without fee provided that copies : are not made or distributed for profit or commercial advantage and that ; copies bear this notice and the full citation on the first page. To copy otherwise, to republish, to post on servers or to redistribute to lists, requires prior specific permission and/or a fee.

F-RAN 5G 2017, July 13-14

Copyright @ 2017 EAI 978-1-63190-156-0 method can reduce the training overhead, the training overhead is still large.

One way of reducing the training overhead is pilot reusing, which will inevitably cause the pilot contamination. In order to mitigate pilot contamination, several works for pilot allocation are proposed [10] [11] [12]. In [11], a smart pilot assignment scheme is proposed to enhance the performance of users with severe pilot contamination. The main idea is to assign the pilot sequence with smallest inter-cell interference to the user having the worst channel quality in a sequential way until all users have been assigned by their corresponding pilot sequences. In [12], the orthogonal training resource allocation problem is proposed for user-centric cooperative network aiming at minimizing the overall training overhead. The optimal solution is found through a graph-theoretic approach. All these methods only consider one channel link, which can not be applied directly in C-RANs due to the different characteristics of radio access link and wireless fronthaul link.

In this paper, a two-phase pilot allocation scheme is proposed to reduce the training overhead in C-RANs. In phase I, the pilot allocation for RRHs is considered. Due to the angular resolution characteristics, the RRH clusters can be formed by whether the channel AoA intervals of the RRHs are nonoverlapping. After the clustering process, the pilot allocation for RRHs can be followed with the principle that the RRHs use orthogonal pilots in intra cluster and reuse pilots in inter clusters. In phase II, a novel pilot allocation algorithm for users is proposed. Firstly, a new metric is presented according to the large-scale coefficients from users to RRHs and the estimated channel matrix from RRHs to BBU pool. Based on the metrics, a user-cluster binary matrix is constructed. Then, an intercluster and intra-cluster pilot allocation scheme is proposed in terms of mitigating the pilot contamination.

\section{SYSTEM MODEL}

Consider a typical cloud radio access network consisting of $M$ users, $K$ RRHs and one BBU pool, where all users and RRHs are equipped with a single antenna, as shown in Fig. 1. For the convenience of layout, the RRHs connect to the BBU pool through the wireless technologies. The channel can be divided into two individual channels, named as radio access link from users to distributed RRHs and wireless fronthaul link from RRHs to the BBU pool. Both channels are assumed to be block fading channel, where channel states stay constant over the coherence block with a length of $\tau$ symbols, and 


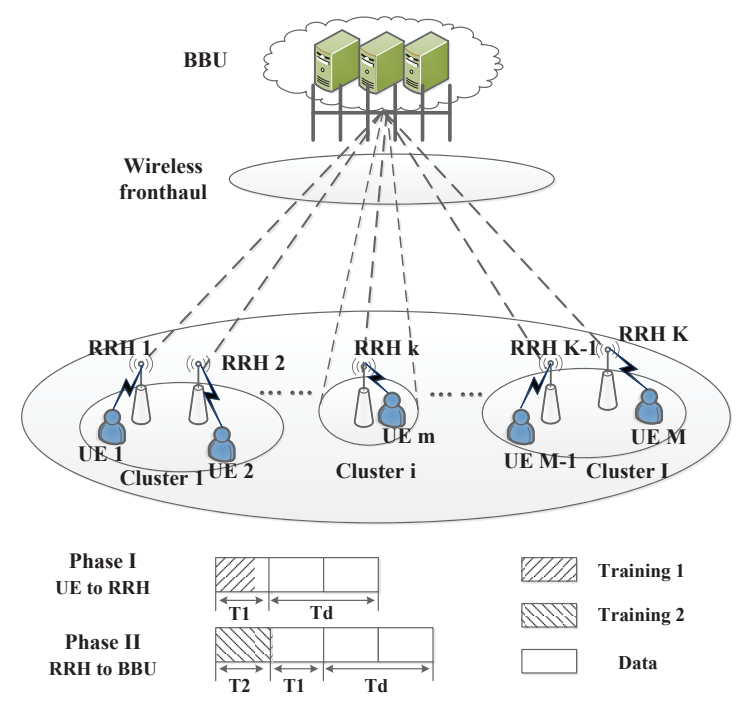

Fig. 1. System model of uplink C-RANs and transmission scheme.

vary from block to block in an independent and identically distributed manner.

\section{A. Channel Model}

Denote the radio access link channel matrix as $\mathbf{H}_{1}$ and wireless fronthaul link channel matrix as $\mathbf{H}_{2}$. The channel matrix $\mathbf{H}_{1}$ is assumed to be spatially uncorrelated, which can be modeled as

$$
\mathbf{H}_{1}=\mathbf{D}^{1 / 2} \odot \mathbf{H}_{\omega},
$$

where $\mathbf{H}_{\omega}$ is a $K \times M$ Gaussian random matrix with independent and identically distributed (i.i.d.) zero mean and unit variance entries. D denotes a $K \times M$ matrix of large scale fading coefficients, which is assumed to be invariant over many coherence time intervals and can be easily obtained by the BBU pool.

For the wireless fronthaul link, $N$ centralized massive antennas are assumed to be deployed at the BBU pool. With the ray-tracing based approach [13], the wireless fronthaul channel between the $N$ antennas at the BBU pool and the antenna of the $k$-th RRH can be modeled as

$$
\mathbf{h}_{2, k}=\frac{1}{\sqrt{P}} \sum_{p=1}^{P} \mathbf{v}\left(\theta_{k p}\right) g_{k p}
$$

where $P(\gg 1)$ is the arbitrary number of i.i.d. paths, $g_{k p} \sim$ $\mathcal{C N}\left(0, \beta_{k}\right)$ is the complex-valued channel gain function and $\mathbf{v}(\theta) \in \mathbb{C}^{N \times 1}$ is the BS array response vector corresponding to the incidence angle $\theta$. Then according to (2), the channel covariance matrix is given by

$$
\mathbf{R}_{2, k}=\mathbb{E}\left\{\mathbf{h}_{2, k} \mathbf{h}_{2, k}^{H}\right\}=\beta_{k} \mathbb{E}\left\{\mathbf{v}\left(\theta_{k}\right) \mathbf{v}\left(\theta_{k}\right)^{H}\right\} .
$$

\section{B. Training Transmission}

The segment training scheme consisting of two phases is adopted in this paper [7], as shown in Fig. 1. During Phase I, the user transmits the pilot sequence $\varphi$ to RRHs. In Phase II, the RRH puts another pilot sequence $\psi$ in the front of $\psi$, and then forwards the re-organized signals to the BBU pool. For the phase I, the received vector $\mathbf{r}_{k}$ at $k$-RRH is given by

$$
\mathbf{r}_{k}=\sum_{m=1}^{M} h_{1, k m} \boldsymbol{\varphi}_{\kappa_{m}}^{T}+\mathbf{n}_{k}^{T},
$$

where $\boldsymbol{\varphi}_{\kappa_{m}} \in \mathbb{C}^{T_{1} \times 1}$ is the pilot vector, $\boldsymbol{\varphi}_{\kappa_{m}}^{H} \boldsymbol{\varphi}_{\kappa_{m}^{\prime}}=$ $T_{1} P_{1} \delta\left(\kappa_{m}-\kappa_{m}^{\prime}\right)$ and $\mathbf{n}_{k}$ is assumed to be independent and identically distributed (i.i.d.) complex Gaussian noise variables with zero-mean and unit variance, i.e. $\mathcal{C N}(0,1)$.

During Phase II, the RRHs put the new pilot sequence $\Psi$ in front of the receive training signals and the total training sequence are transmitted to the BBU pool. The received training signals at the BBU pool can be divided into two parts:

$$
\mathbf{Y}_{2}=\mathbf{H}_{2} \boldsymbol{\Psi}+\mathbf{N}_{2}=\sum_{k=1}^{K} \mathbf{h}_{2, k} \boldsymbol{\psi}_{\pi_{k}}^{T}+\mathbf{N}_{2}
$$

$$
\mathbf{Y}_{1}=\sum_{k=1}^{K} \mathbf{h}_{2, k}\left(\sum_{m=1}^{M} h_{1, k m} \boldsymbol{\varphi}_{\kappa_{m}}^{T}+\mathbf{n}_{k}^{T}\right)+\mathbf{N}_{1}
$$

where $\mathbf{H}_{2}=\left[\mathbf{h}_{2,1}, \mathbf{h}_{2,2}, \ldots, \mathbf{h}_{2, K}\right]$ and $\mathbf{\Psi}=$ $\left[\boldsymbol{\psi}_{\pi_{1}}, \boldsymbol{\psi}_{\pi_{2}}, \ldots, \boldsymbol{\psi}_{\pi_{K}}\right]^{T}, \quad \boldsymbol{\psi}_{\pi_{m}}^{H} \boldsymbol{\psi}_{\pi_{m}^{\prime}}=\tau_{2} P_{2} \delta\left(\pi_{m}-\pi_{m}^{\prime}\right)$. $\mathbf{N}_{1}$ and $\mathbf{N}_{2}$ are assumed to be independent and identically distributed (i.i.d.) complex Gaussian noise variables with zero-mean and unit variance, i.e. $\mathcal{C N}(0,1)$.

\section{Two Phase based Pilot Allocation Scheme}

To enhance the estimation performance, one straightforward way is to assign each RRH and each user an orthogonal training resource, whose overall training overhead increases linearly with the total number of RRHs and users. However, it is impossible for the realistic systems since the number of RRHs and users are large in C-RANs. Allocating more symbols for training will degrade the system spectral efficiency due to the limited channel coherence time. Another choice is to reuse the pilots among clusters, which will inevitably cause the pilot contamination. To mitigate this phenomenon, pilot allocation scheme is needed.

\section{A. Pilot allocation during Phase I}

We first discuss the pilot allocation for RRHs and the corresponding channel estimation error.

Assume that the index sets of the RRHs and users are denoted as $\mathcal{K}=\{1,2, \ldots, K\}$ and $\mathcal{M}=\{1,2, \ldots, M\}$, respectively. The total training interval length equals to $T$, where the training interval length for users and RRHs are $T_{1}$ and $T_{2}$, respectively. The available orthogonal pilot set for users is $\mathcal{T}_{1}=\left\{1,2, \ldots, T_{1}\right\}$ and that for RRHs is $\mathcal{T}_{2}=\left\{1,2, \ldots, T_{2}\right\}$. The set of clusters as $C_{i}, i=1,2, \ldots, I$ and the set of RRHs and users in cluster $C_{i}$ as $\mathcal{K}_{i}$ and $\mathcal{M}_{i}, \sum_{i=1}^{I}\left|\mathcal{K}_{i}\right|=K$, $\sum_{i=1}^{I}\left|\mathcal{M}_{i}\right|=M$.

Denote $\mathcal{K}_{\kappa_{k}}$ as the set of the RRHs using the same pilot sequence as $k$-th RRH and the pilot sequence $\boldsymbol{\psi}_{\kappa_{k}}$ is allocated 
to the $k$-th RRH. Then the MMSE estimate of the channel $\mathbf{h}_{2, k}$ is given by [13] [14]

$$
\hat{\mathbf{h}}_{2, k}=\mathbf{R}_{2, k} \mathbf{C}_{\kappa_{k}}^{-1} \mathbf{y}_{\pi_{k}}
$$

where

$$
\begin{gathered}
\mathbf{C}_{\kappa_{k}}=\sum_{l \in \mathcal{K}_{\kappa_{k}}} \mathbf{R}_{l}+\frac{1}{P_{2} T_{2}} \mathbf{I}, \\
\mathbf{y}_{\kappa_{k}}=\sum_{l \in \mathcal{K}_{\kappa_{k}}} \mathbf{h}_{2, l}+\frac{1}{P_{2} T_{2}} \mathbf{N}_{2} \boldsymbol{\psi}_{\kappa_{k}}^{*} .
\end{gathered}
$$

The channel estimation error covariance is

$$
\mathbf{R}_{\widetilde{\mathbf{h}}_{2, k}}=\mathbf{R}_{2, k}-\mathbf{R}_{2, k} \mathbf{C}_{\kappa_{k}}^{-1} \mathbf{R}_{2, k},
$$

so the total MSE of $\mathbf{H}_{2}$ is

$$
\epsilon=\sum_{k=1}^{K} \operatorname{Tr}\left\{\mathbf{R}_{\widetilde{\mathbf{h}}_{2, \mathrm{k}}}\right\} .
$$

According to the Theorem 1 in [13], if the RRHs reusing the pilots have non-overlapping channel AoA intervals, i.e. for $\forall i, j \in \mathcal{K}$ and $i \neq j$,

$$
\theta\left(\mathbf{R}_{2, i}, \mathbf{R}_{2, j}\right)=\frac{\pi}{2} \text {, when } \kappa_{i}=\kappa_{j},
$$

the minimum value of the MSE can be achieved, which is given by

$$
\epsilon=\sum_{k=1}^{K} \operatorname{Tr}\left\{\mathbf{R}_{2, k}-\mathbf{R}_{2, k}\left(\mathbf{R}_{2, k}+\frac{1}{P_{2} T_{2}} \mathbf{I}\right)^{-1} \mathbf{R}_{2, k}\right\} .
$$

Due to the fact that the BS is required to be elevated at high altitude, such as at the top of high building, there are few surrounding scatterers at the end of BS, which indicates the incident angles of the RRH is usually limited in the narrow angular range. Therefore, the clusters of RRHs can be formed depending on whether the channel AoA intervals of the RRHs are non-overlapping. If channel AoA intervals of the UTs reusing the pilots are non-overlapping, the pilot interference does not take in effect. The wireless fronthaul link channel can be estimated with little pilot contamination by assigning the orthogonal training in each cluster and reusing the training among clusters.

Since the cluster of RRHs are formed according to the AoA intervals, it can be ensured that the reusing pilots in RRHs have little impact on the estimation performance. The length of training assigned for RRH can be reduced from $K$ to $T_{2}$. $T_{2}$ only needs to satisfy $T_{2} \geq \max _{i}\left|\mathcal{K}_{i}\right|$, which means the training overhead of RRHs can be reduced greatly.

In this paper, it is assumed that the channel covariance matrices can be obtained by the BBU pool. Although channel covariance matrices vary over time, it changes less frequently compared to the instantaneous CSI and can stay constant over a wide frequency interval. Thus, we can estimate the channel covariance matrices through averaging over frequency in practical C-RANs.

\section{B. Pilot allocation during Phase II}

In this subsection, the pilot allocation scheme for users is discussed to make the total MSE minimization. The estimation of radio access link channel $\mathbf{H}_{1}$ is followed by the estimation of wireless fronthaul link channel $\mathbf{H}_{2}$. For the convenience of calculation, the channel coefficients of $\mathbf{H}_{2}$ is assumed to be perfect and the estimation error is omitted here.

To estimate the channel $\mathbf{H}_{1}$, we first right multiply $\frac{1}{T_{1} P_{1}} \boldsymbol{\varphi}_{\pi_{j}}^{*}$ on equation (6) as

$$
\begin{aligned}
\mathbf{y}_{\pi_{j}} & \triangleq \frac{1}{T_{1} P_{1}} \mathbf{Y}_{1} \boldsymbol{\varphi}_{\pi_{j}}^{*} \\
& =\sum_{k=1}^{K} \mathbf{h}_{2, k} \sum_{m \in \mathcal{M}_{\pi_{j}}} h_{1, k m}+\frac{1}{T_{1} P_{1}} \sum_{k=1}^{K} \mathbf{h}_{2, k} \mathbf{n}_{k}^{T} \boldsymbol{\varphi}_{\pi_{j}}^{*} \\
& +\frac{1}{T_{1} P_{1}} \mathbf{N}_{1} \boldsymbol{\varphi}_{\pi_{j}}^{*},
\end{aligned}
$$

where $\mathcal{M}_{\pi_{j}}$ is the set of users using the same pilot sequence as $j$-th user.

Without loss of generality, we focus on a typical cluster $C_{i}$ with $K_{i}$ RRHs and $M_{i}$ users. Then the equation above can be written as

$$
\begin{aligned}
\mathbf{y}_{\pi_{j}} & =\sum_{k \in C_{i}} \mathbf{h}_{2, k} h_{1, k j}+\sum_{k \in C_{i}} \mathbf{h}_{2, k} \sum_{m \in \mathcal{M}_{\pi_{j}} \backslash\{j\}} h_{1, k m} \\
& +\sum_{k \in \mathcal{K} \backslash C_{i}} \mathbf{h}_{2, k} \sum_{m \in \mathcal{M}_{\pi_{j}}} h_{1, k m}+\frac{1}{T_{1} P_{1}} \sum_{k=1}^{K} \mathbf{h}_{2, k} \mathbf{n}_{k}^{T} \boldsymbol{\varphi}_{\pi_{j}}^{*} \\
& +\frac{1}{T_{1} P_{1}} \mathbf{N}_{1} \boldsymbol{\varphi}_{\pi_{j}}^{*},
\end{aligned}
$$

where the second term is the inter-cluster interference from the user using the same training sequence in other clusters and the third term is the inter-cluster interference from the RRHs which forward the same training sequence in other clusters.

Denote that $\mathbf{H}_{2, C_{i}}=\left[\mathbf{h}_{2,1}^{C_{i}}, \mathbf{h}_{2,2}^{C_{i}}, \ldots, \mathbf{h}_{2, K_{i}}^{C_{i}}\right]$ and $\mathbf{h}_{1, j}^{C_{i}}=$ $\left[h_{1,1 j}^{C_{i}}, h_{1,2 j}^{C_{i}}, \ldots, h_{1, K_{i} j}^{C_{i}}\right]^{T}$, then the estimation of $\mathbf{h}_{1, j}^{C_{i}}$ can be expressed as

$$
\widehat{\mathbf{h}}_{1, j}^{C_{i}}=\mathbf{D}_{j}^{C_{i}} \mathbf{H}_{2, C_{i}}^{H}\left(\sum_{m \in \mathcal{M}_{\pi_{j}}} \mathbf{H}_{2} \mathbf{D}_{m} \mathbf{H}_{2}^{H}+\mathbf{S}\right)^{-1} \mathbf{y}_{\pi_{j}},
$$

where $\mathbf{S}=\frac{1}{\tau_{1} P_{1}} \sum_{k=1}^{K} \mathbf{R}_{2, k}+\frac{1}{\tau_{1} P_{1}} \mathbf{I}$ and $\mathbf{D}_{m}=$ $\operatorname{diag}\left(\lambda_{1 m}, \lambda_{2 m}, \ldots, \lambda_{K m}\right)$.

The corresponding MSE can be given at the top of next page.

Then, the problem of pilot allocation scheme for users with the target of minimizing the total MSE can be formulated as

$$
\min _{\mathcal{P}\left(\mathcal{M}, \mathcal{T}_{1}\right)} \mathrm{MSE}=\sum_{i=1}^{I} \sum_{j=1}^{M} \mathrm{MSE}_{C_{i}, j} .
$$

It is a combinatorial optimization problem, and the optimal pilot allocation can be derived through exhaustive search. However, it is hard to implement in practice because of the high complexity.

From equation (20), it is shown that the MSE of $j$-th user for a typical cluster $C_{i}$ is affected by $\sum_{k \in C_{i}}\left(\lambda_{k j}^{C_{i}}\right)^{2} \mathbf{h}_{2, k}^{C_{i}}\left(\mathbf{h}_{2, k}^{C_{i}}\right)^{H}$ 


$$
\operatorname{MSE}_{C_{i}, j}=\operatorname{Tr}\left(\mathbf{D}_{j}^{C_{i}}\right)-\operatorname{Tr}\left[\sum_{k \in C_{i}}\left(\lambda_{k j}^{C_{i}}\right)^{2} \mathbf{h}_{2, k}^{C_{i}}\left(\mathbf{h}_{2, k}^{C_{i}}\right)^{H}\left(\sum_{m \in \mathcal{M}_{\pi_{j}}} \mathbf{H}_{2} \mathbf{D}_{m} \mathbf{H}_{2}^{H}+\mathbf{S}\right)^{-1}\right]
$$

and the other users reusing the same pilot as $j$-th user, i.e. $\sum_{m \in \mathcal{M}_{\pi_{j}}} \mathbf{H}_{2} \mathbf{D}_{m} \mathbf{H}_{2}^{H}$. Thus, we first introduce $o_{j}^{C_{i}}=$ $\operatorname{Tr}\left(\sum_{k \in C_{i}}\left(\lambda_{k j}^{C_{i}}\right)^{2} \mathbf{h}_{2, k}^{C_{i}}\left(\mathbf{h}_{2, k}^{C_{i}}\right)^{H}\right)$ as the metric which means $j$ th user is "close" to the cluster $C_{i}$. Since the large-scale fading coefficients $\lambda_{k j}^{C_{i}}$ is known to the BBU pool and the channel matrix $\mathbf{H}_{2}$ is estimated previously, this metric can be easily obtained at the BBU pool. Then, we sort $o_{j}^{C_{i}}$ in descending order for cluster $C_{i}$, i.e.

$$
\left[o_{\chi_{1}}^{C_{i}}, o_{\chi_{2}}^{C_{i}}, \ldots, o_{\chi_{M}}^{C_{i}}\right], i=1,2, \ldots, I
$$

where $\chi_{1}, \chi_{2}, \ldots, \chi_{M}$ is the permutation and combination of users. It is obvious that the first $M_{i}$ metrics represent the users in the $C_{i}$ cluster and the last few metrics indicant the users far away from the $C_{i}$ cluster whose pilot contamination influence can be ignored. Dislike the traditional centralized MIMO systems, the user in the center may not has the most valuable metric. Instead, it is decided by both large-scale coefficients from users to RRHs and the channel matrix coefficients from RRHs to BBU pool.

The main idea of our scheme is that the users which have the same level value of $o_{j}^{C_{i}}$ have the priority to allocate the orthogonal pilots. In particular, the same level is defined as greater than or equal to the value of $\eta o_{\chi_{M_{i}}}^{C_{i}}$ and less than or equal to $o_{\chi_{M_{i}}}^{C_{i}}$, where $0<\eta<1$. Then, the user-cluster binary matrix $\mathbf{F} \in \mathcal{R}^{M \times I}$ can be derived, whose element is defined as

$$
f_{j i}= \begin{cases}1 & \text { if } \eta o_{M_{i}}^{C_{i}} \leq o_{j}^{C_{i}} \leq o_{M_{i}}^{C_{i}}, \\ 0 & \text { else, }\end{cases}
$$

where $f_{j i}=1$ indicates that $j$-user outside the $i$-th cluster has the impact on the $i$-th cluster.

After the definition of user-cluster binary matrix, the influence of user to each cluster can be derived intuitively. Defining $\mathbf{f}_{j}$ is the $j$-th row of the matrix, we calculate the value of $\mathbf{f}_{i} \mathbf{f}_{j}^{T}$. Generally, this process should have $(M+1) M / 2$ times. If $\mathbf{f}_{i} \mathbf{f}_{j}^{T}$ is not equal to 0 , i.e. $\mathbf{f}_{j} \mathbf{f}_{i}^{T} \neq 0, i \neq j$, it means that $i$-th user and $j$-th user have the same level influence to some cluster, which indicates that these two users should be assigned orthogonal training. This process can be set as intercluster pilot allocation.

After the inter-cluster pilot allocation, the remaining users which has not been assigned a pilot are those which has the most valuable metrics in their corresponding cluster and has less influence on the adjacent clusters. For the pilot allocation of these users, the principle is that the users in the cluster can not be reused. This process can be set as intra-cluster pilot allocation.

The above process can be summarized as Algorithm 1.

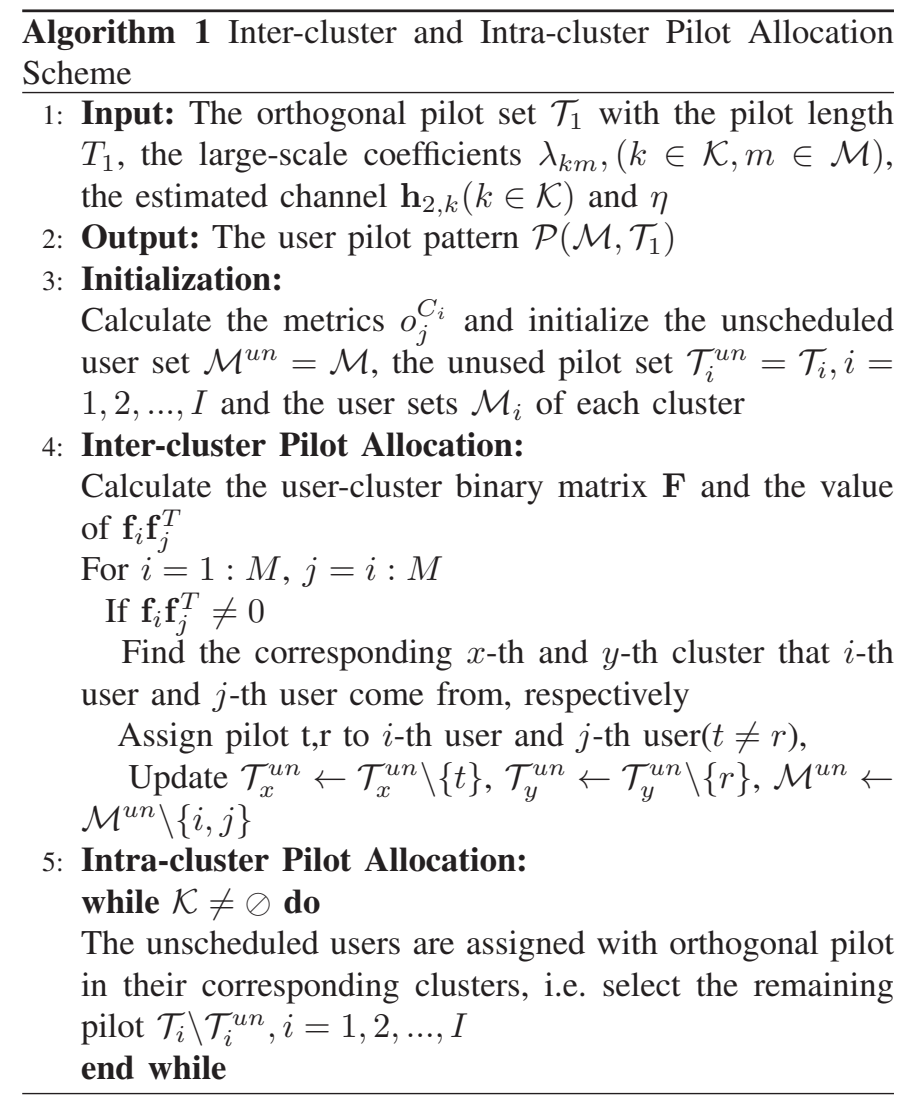

\section{Simulation Results}

In this section, the simulation results are presented to evaluate the performance of our proposed algorithms. We first assume that the BBU pool is equipped with $N=128$ ULA antenna and the antennas are spaced with a half wavelength distance. The power for RRHs and UEs are set to be equal, i.e. $P_{1}=P_{2}=P$ and the noise variance is set to be one. The path loss exponent is set as $\alpha=3.8$. We consider the case that $K=8$ and $M=8$ and the mean channel AoAs of the RRHs from RRH 1 to RRH 8 are [0.9834 - 0.3651 $0.40121 .0134-0.32140 .23780 .19960 .9523]$ in radians. The AoAs are uniformly distributed over $\left[\bar{\theta}_{k}-\Delta \theta, \bar{\theta}_{k}+\Delta \theta\right]$, where $\bar{\theta}_{k}$ is the mean AoA. Therefore, the RRH can be formed into three clusters, where the RRH index of cluster $1,2,3$ are $\{1,4,8\},\{2,3,5\},\{6,7\}$, respectively.

In Fig. 2, the normalized MSE of $\mathbf{H}_{2}$ versus SNR for the different pilot allocation schemes is evaluated. Three schemes including random pilot allocation, reusing pilot allocation and orthogonal pilot allocation schemes are compared. The length of pilot for random pilot scheme and that for orthogonal pilot scheme is 8 . The length of proposed scheme varies from 3 to 


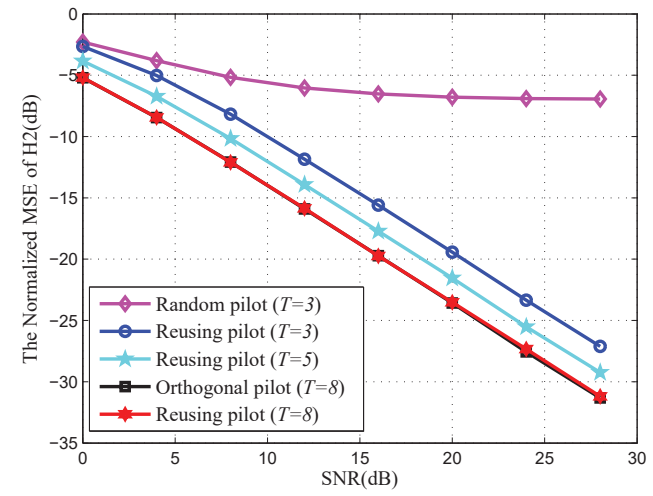

Fig. 2. The normalized MSE comparison for different pilot allocation schemes of $\mathbf{H}_{2}$.

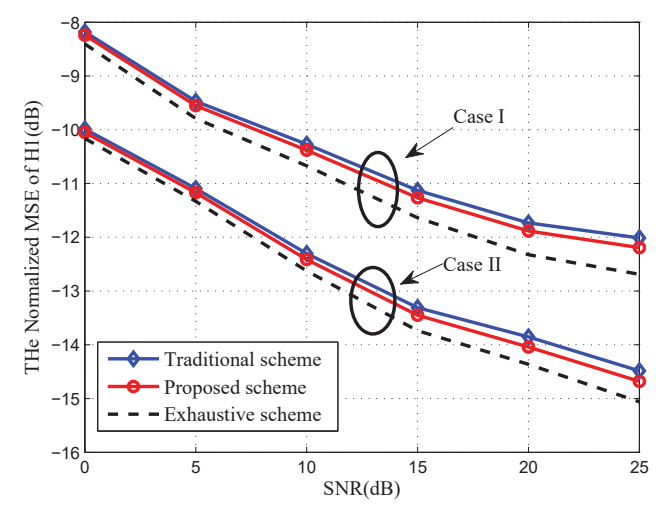

Fig. 3. The normalized MSE comparison for different pilot allocation schemes of $\mathbf{H}_{1}$.

8. It can be observed that the MSEs of both the reusing pilot scheme and orthogonal pilot scheme are decaying steeply to zero when the SNR increases. Meanwhile, the MSE of random scheme converging to non-zero error floors. This is due to the pilot contamination caused by the random pilot allocation for RRHs and the reusing pilot scheme can sufficiently mitigate this contamination. When the length of proposed scheme trends to 8, the MSE of proposed scheme almost coincides with the orthogonal scheme. However, in this case, only 3 pilots are adopted in our proposed scheme compared to 8 pilot used in the orthogonal scheme.

Fig. 3 presents the normalized MSE of $\mathbf{H}_{1}$ versus SNR, where exhaustive scheme, traditional scheme and our proposed scheme are considered. The length of pilot are set to 3 . Two cases are considered including severe inter-cluster interference in Case I and slight one in Case II. It can be seen that the performance gap of the three scheme are small in Case II because in the sight inter-cluster interference case, the users have little impact on the users in other adjacent cluster. Meanwhile, in Case I, the performance of the proposed scheme is better than the traditional scheme during whole SNR region with a little bit improvement, which verifies the effectiveness of the proposed scheme.

\section{CONCLUSIONS}

In this paper, the pilot allocation scheme for both fronthaul and access links are considered in C-RANs in order to reduce the training overhead and mitigate pilot contamination. The pilot allocation scheme for fronthaul link is developed due to the high angular resolution characteristic, where one RRH has little impact on another RRH if their AoA are non-overlapping. For the access link, an inter-cluster and intra-cluster pilot allocation scheme is proposed via the metric defined according to the large scale coefficients from users to RRHs and the estimated fronthaul channel matrix. Numerical results have shown that the proposed schemes can achieve considerable performance effectively.

\section{ACKNOWLEDGMENT}

This work was supported National Natural Science Foundation of China (Grant No. 61501045) and the State Major Scienceand Technology Special Projects (Grant No. 2016ZX03001017-004).

\section{REFERENCES}

[1] D. Wu, J. He, H. Wang, C. Wang and R. Wang, "A hierarchical packet forwarding mechanism for energy harvesting wireless sensor networks," IEEE Commun. Mag., vol. 53, no. 8, pp. 92-98, Aug. 2015.

[2] D. Wu, Y. Wang, H. Wang, B. Yang, C. Wang, R. Wang, "Dynamic coding control in social intermittent connectivity wireless networks," IEEE Trans. Veh. Technol., vol 65, no. 9, pp. 7634-7646, Oct. 2016.

[3] D. Wu, P. Zhang, H. Wang, C. Wang, R. Wang, "Node service ability aware packet forwarding mechanism in intermittently connected wireless networks," IEEE Trans. Wireless Commun., vol. 15, no. 12, pp. 81698181, Sep. 2016.

[4] M. Peng, Y. Li, Z. Zhao and C. Wang, "System architecture and key technologies for $5 \mathrm{G}$ heterogeneous cloud radio access networks," IEEE Network., vol. 29, no. 2, pp. 6-14, Mar. 2015.

[5] Z. Zhao, M. Peng, Z. Ding, W. Wang, and H. V. Poor, "Cluster content caching: An energy-efficient approach to improve quality of service in cloud radio access networks," IEEE J. Sel. Areas Commun., vol. 34, no. 5, pp. 1207-1221, May 2016.

[6] Z. Zhao, M. Peng, Z. Ding, C.Wang, and H. V. Poor, "Cluster formation in cloud-radio access networks: Performance analysis and algorithms design," in Proc. IEEE ICC 2015, London, UK, June 2015

[7] Q. Hu, M. Peng, X. Xie, Z. Mao and H. V. Poor, "Training design for channel estimation in uplink cloud radio access networks," IEEE Trans. Signal Process., vol. 22, no. 8, pp. 1060-1064, Aug. 2015.

[8] X. Xie, M. Peng, W. Wang and H. V. Poor, "Training design and channel estimation in uplink cloud radio access networks," IEEE Signal Process. Lett., vol. 22, no. 8, pp. 1060-1064, Aug. 2015.

[9] Z. Zhao, Y. Ban, D. Chen, Z. Mao, and Y. Li, "Joint design of iterative training-based channel estimation and cluster formation in cloud-radio access networks," IEEE Access., vol. 4, pp. 9643-9658, Oct. 2016.

[10] X. Zhu, L. Dai and Z. Wang, "Graph coloring based pilot allocation to mitigate pilot contamination for multi-cell massive MIMO systems," IEEE Commun. Lett., vol. 19, no. 10, pp. 1842-1845, Oct. 2015.

[11] X. Zhu, Z. Wang, L. Dai and C. Qian, "Smart pilot assignment for massive MIMO," IEEE Commun. Lett., vol. 19, no. 9, pp. 1644-1647, Sep. 2015

[12] Z. Chen, X. Hou and C. Yang, "Training resource allocation for usercentric base station cooperation networks," IEEE Trans. Veh. Technol., vol. 65, no. 4, pp. 2729-2735, Apr. 2016.

[13] L. You, X. Gao, X. Xia, N. Ma and Y. Peng, "Pilot reuse for massive MIMO transmission over spatially correlated Rayleigh fading channels," IEEE Trans. Wireless Commun., vol. 14, no. 6, pp. 3352-3366, Jun. 2015.

[14] S. M. Kay, "Fundamentals of statistical signal processing: estimation theory." Englewood Cliffs, NJ: Prentice Hall, 1993. 